\title{
Management of occluded biliary Wallstents
}

T C K Tham, D L Carr-Locke, J Vandervoort, R C K Wong, D R Lichtenstein, J Van Dam, F Ruymann, S Chow, J J Bosco, T Qaseem, D Howell, D Pleskow, W Vannerman, E D Libby

\begin{abstract}
Background-Wallstents (Schneider Stent, Inc., USA) used for the palliation of malignant biliary strictures, although associated with prolonged patency, can occlude. There is no consensus regarding the optimal management of Wallstent occlusion.

Aims-To evaluate the efficacy of different endoscopic methods for managing biliary Wallstent occlusion.

Methods-A multicentre retrospective study of patients managed for a biliary Wallstent occlusion.

Results-Data were available for 38 patients with 44 Wallstent occlusions, all of which had initial endoscopic management. Twenty four patients had died and 14 were alive after a median follow up of 231 (30-1095) days following Wallstent occlusion. Occlusions were managed by insertion of another Wallstent in 19, insertion of a plastic stent in 20 , and mechanical cleaning in five. Endoscopic management was successful in $43(98 \%)$. Following management of the occlusion, bilirubin decreased from $6.0(0.5-34.3)$ to $2.1(0.2-27.7) \quad \mathrm{mg} / 100 \mathrm{ml}(\mathrm{p}<0.05)$. No complications occurred. The median duration of second stent patency was 75 days (95\% confidence interval 43 to 107$)$ after insertion of another Wallstent, 90 days (71 to 109) after insertion of a plastic stent, and 34 days (30 to 38 ) after mechanical cleaning (NS). The respective median survivals were 70 days (22-118), 98 days (54-142), and 34 days (30-380) (NS). Incremental cost effective analysis showed that plastic stent insertion is the most cost effective option.
\end{abstract}

Conclusion-Although all three methods are equally effective in managing an occluded Wallstent, the most cost effective method appears to be plastic stent insertion.

(Gut 1998;42:703-707)

Keywords: self expanding metal stent; biliary strictures; stent occlusion; jaundice; gastrointestinal malignancy

Winchester Hospital, MA, USA

W Vannerman

New England Medical Center, MA, USA

E D Libby

Correspondence to: Dr T C K Tham, Ulster Hospital, Dundonald, Belfast BT16 0RH, Northern Ireland, UK.

Accepted for publication 26 November 1997
Wallstent is a tubular stainless steel uncovered super alloy mesh delivered in a constrained form on an 8 or 7.5 French gauge catheter system which, when deployed, expands to a final diameter of 24 or 30 French gauge ( 8 or 10
Self expanding metallic stents such as the allstent (Schneider Stent Inc., Minneapolis, Minnesota, USA) have been used for the management of biliary strictures to provide perma- $\mathrm{mm}$ ) and shortens to a length of 42,68 , or 80 $\mathrm{mm}$. The stent is deployed using the Unistep system which allows easy retraction of the covering membrane after internal wetting of its inner hydrophilic coating.

The main advantage of Wallstents over plastic stents for the palliation of malignant biliary obstruction is that they have a longer patency and despite the initial cost, have been shown to be cost effective. ${ }^{12}$ Wallstents still occlude after a median interval of nine months ${ }^{12}$ but unlike plastic stents, they cannot be extracted. Several methods have been used in the management of the occlusion such as insertion of another Wallstent or plastic stent, or mechanical cleaning. There are currently no data comparing the efficacy of the different management options and follow up of the Wallstent occlusion. Such data would be useful in deciding on optimal management.

\section{Methods}

A multicentre retrospective study of six biliary endoscopy centres in New England, USA, was undertaken to identify patients who were treated for biliary Wallstent occlusion. Detailed questionnaires were completed for each patient managed for a biliary Wallstent occlusion by the endoscopist involved.

All primary Wallstents were correctly deployed across a biliary stricture to allow bile drainage. Tumour ingrowth was assumed when cholangiography showed a tight stricture within the stent, the appearance of which was similar to the original malignant stricture, and passage of a diagnostic catheter was difficult (fig 1). Tumour overgrowth was assumed when cholangiography showed a new stricture proximal or distal to the stent (fig 2). Debris or sludge occlusion was diagnosed when cholangiography showed filling defects within the lumen of the stent and further instrumentation showed passage of debris through the distal portion of the stent confirmed endoscopically (fig 3). When the Wallstents became occluded, they were managed either by insertion of another Wallstent within the first (fig 4), a plastic stent within the first Wallstent (fig 5), or mechanical cleaning of the Wallstent (fig 3). Mechanical cleaning was defined as passage of an instrument (balloon, catheter, or guidewire) to allow recanalisation of the Wallstent to allow biliary drainage. Plastic stents were either the curved 10 French gauge Cotton-Leung stents (in $86 \%$ ) or the straight Amsterdam stents (in 14\%) (Wilson-Cook Medical Inc., WinstonSalem, North Carolina, USA). Five patients $(24 \%)$ had two plastic stents inserted during the same procedure (fig 5). 


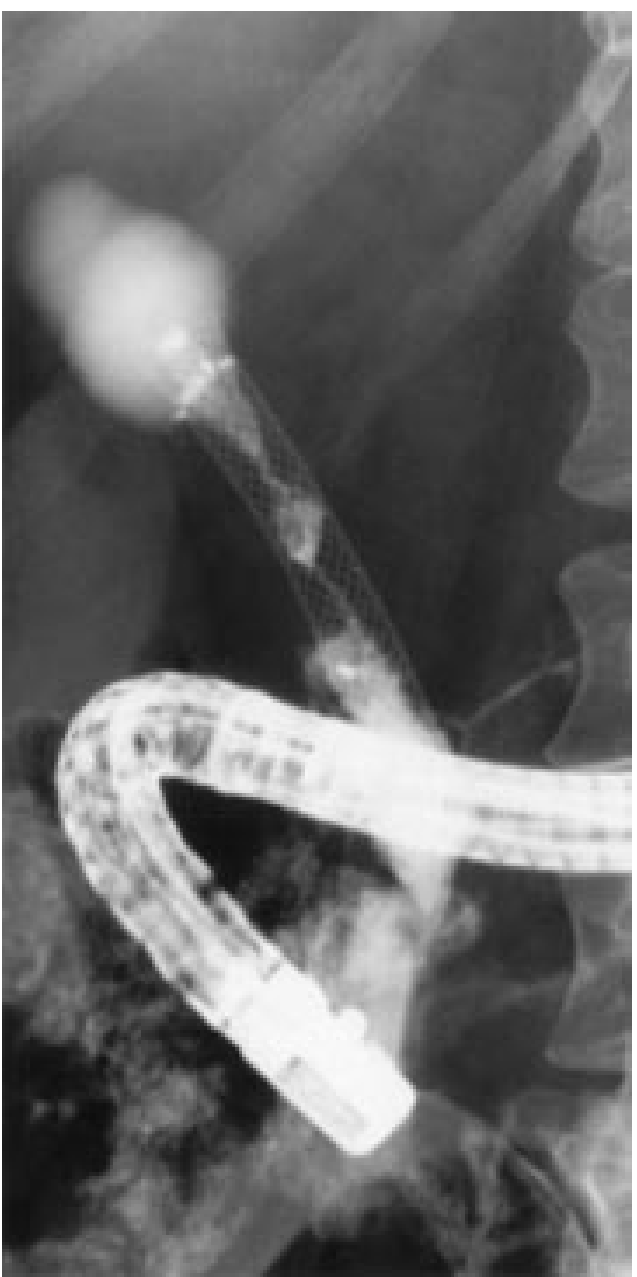

Figure 1 Tumour ingrowth within a Wallstent.

Follow up data were obtained by the physician managing the Wallstent occlusion from the patient's medical record and/or by contacting their primary care physician. Management of the Wallstent occlusion was considered successful if there was clinical improvement with a significant fall in bilirubin following intervention. Complications of endoscopic retrograde cholangiopancreatography (ERCP) were defined according to published criteria. ${ }^{3}$

Second stent patency represented the interval between the time of treatment of the Wallstent occlusion and the time of its reocclusion or the death of the patient with jaundice and fever. Survival represented the interval between the time of treatment of the Wallstent occlusion and the patient's death. Death without jaundice or sepsis was assumed to be due to causes other than stent occlusion.

An incremental cost effectiveness analysis was done as described by Detsky and Naglie. ${ }^{4}$ The cost for all treatment strategies differed only with respect to equipment price. The cost was $\$ 995$ ( $£ 662$ ) for a Wallstent, $\$ 83(£ 52)$ for a plastic stent $(\$ 33(£ 21)$ for plastic stent and $\$ 50(£ 31)$ for a disposable stent insertion system; OASIS, Wilson-Cook), and $\$ 115$ for a balloon used for mechanical cleaning. Effectiveness was expressed as mean number of pro-

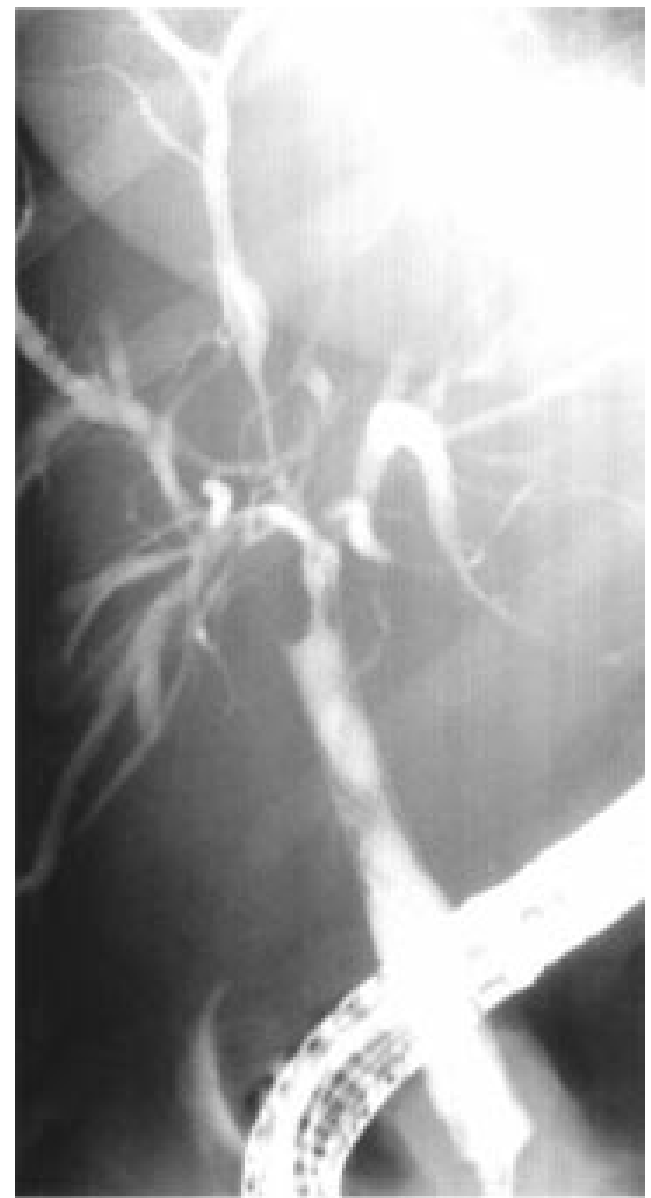

Figure 2 Tumour overgrowth proximal to a Wallstent.

cedures necessary for each treatment strategy until death or the end of evaluation.

Cumulative stent patency and patient survival were estimated according to the KaplanMeier technique supplemented by the log rank test for comparison between groups. Bilirubin concentrations before and after treatment were analysed using the Kruskal-Wallis test. A p value of less than 0.05 was considered significant. Correlation between the period of patency of the first Wallstent and the period of patency of the second procedure was analysed using regression analysis.

\section{Results}

A total of 152 Wallstents had been inserted endoscopically in the six centres. Of these, 34 Wallstents (22\%) occluded. Five other patients were treated for occluded Wallstents which had originally been inserted percutaneously. Data were available for 38 patients with 44 Wallstent occlusions. Twenty three of the $38(61 \%)$ had prior occluded plastic stents. The number of Wallstents inserted which became occluded were: one Wallstent in 33 patients, two Wallstents in four patients; and three Wallstents in one patient. Indications for the Wallstents were: pancreatic carcinoma in 14, metastatic carcinoma in 10 , cholangiocarcinoma in nine, lymphoma in two, gall bladder carcinoma in one, duodenal carcinoma in one, and benign postoperative stricture in one. 

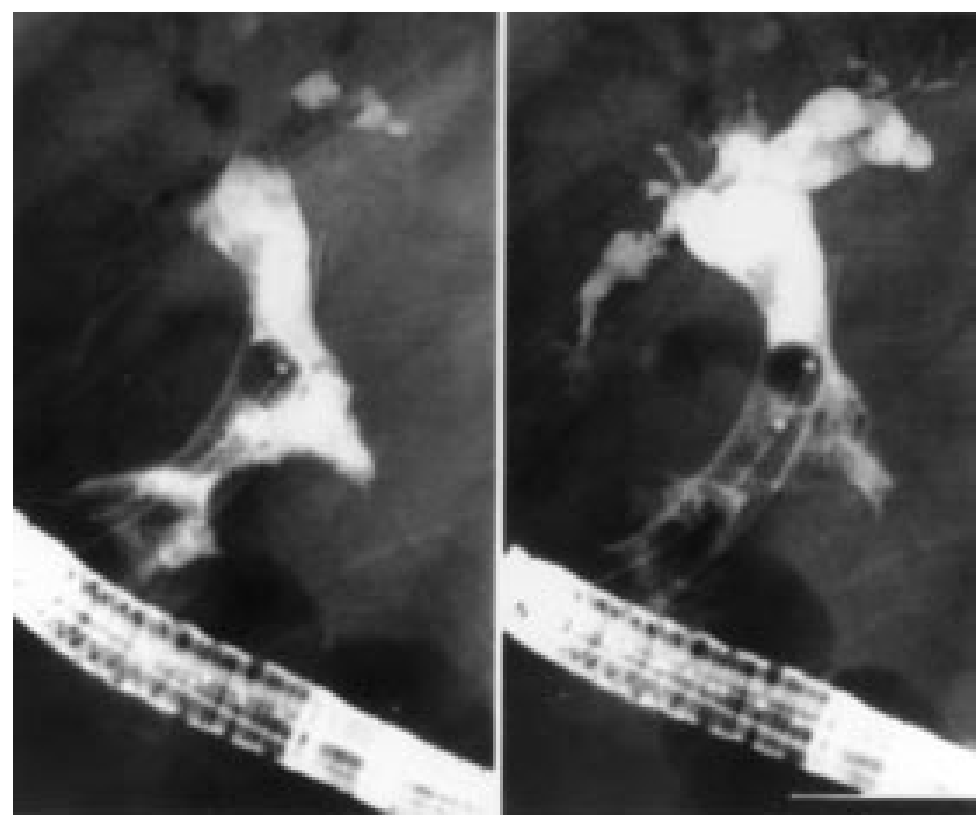

Figure 3 Debris within a Wallstent causing an obstruction. The debris was extracted using a balloon (mechanical cleaning).

Median duration of first Wallstent patency was 102 days (range 12-280). Wallstent occlusions presented as: cholangitis in 25 , jaundice in 11 , abnormal liver function tests in six, "smouldering pancreatitis" in one, and was unspecified in one. The cause of Wallstent occlusion was: tumour ingrowth in 19, tumour ingrowth and overgrowth in nine, tumour overgrowth only in three, debris in eight, "hyperplasia" in two (one in benign stricture), and was undefined in two.

All patients had initial endoscopic management of their Wallstent occlusion which was successful in 43 occlusions (98\%). One failure was in a patient with a hilar tumour where endoscopic insertion of a plastic stent in the left hepatic duct did not resolve her jaundice and subsequently proceeded to successful percutaneous insertion of a plastic stent in the right hepatic duct. Another patient who had a partial

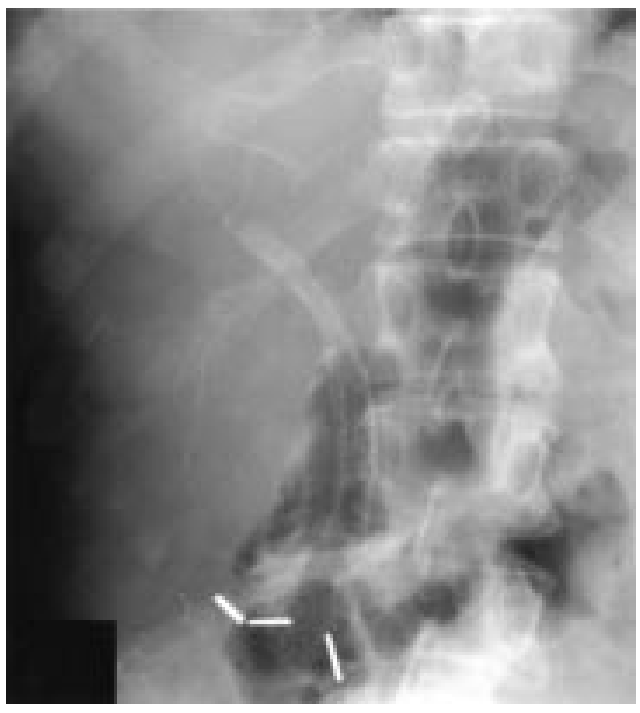

Figure 4 Second Wallstent inserted for occlusion of the first Wallstent showing good patency after deployment. benefit from an endoscopically placed plastic stent in the right hepatic duct proceeded to successful endoscopic insertion of a plastic stent into the left hepatic duct. One patient who had a plastic stent inserted proceeded to have an elective stent exchange after 30 days. The remainder of the patients who had plastic stents inserted within the Wallstent had plastic stent exchange only if they became symptomatic.

No significant complications occurred following management of Wallstent occlusion by any method. Following management of the occlusion, bilirubin decreased from a median of $6.0(0.5-34.3)$ to $2.1(0.2-27.7) \mathrm{mg} / 100 \mathrm{ml}$ $(\mathrm{p}<0.05)$.

The median follow up was 75 days (range 14-353) following insertion of another Wallstent, 128 days (7-370) following insertion of a plastic stent, and 176 days (9-278) following mechanical cleaning. At the time of evaluation, $12 / 19$ patients $(63 \%)$ who had insertion of another Wallstent, 11/20 (55\%) who had insertion of a plastic stent, and $3 / 5(60 \%)$ who had mechanical cleaning had died. Median survival (fig 6) after management of the initial Wallstent occlusion was 70 days $(95 \%$ confidence interval 22 to 118 ), 98 days (54 to 142 ), and 34 days (30 to 38 ) respectively. There was no statistical difference in survival between the different treatments.

Stent occlusion recurred following management of the initial Wallstent occlusion in six patients $(32 \%)$ who had another Wallstent inserted, five patients (25\%) who had a plastic stent inserted, and three patients $(60 \%)$ following mechanical cleaning. The median stent patency (fig 7) following management of the primary Wallstent occlusion was 75 days (95\% confidence interval 43 to 107), 90 days (71 to 109$)$, and 34 days (30 to 38 )

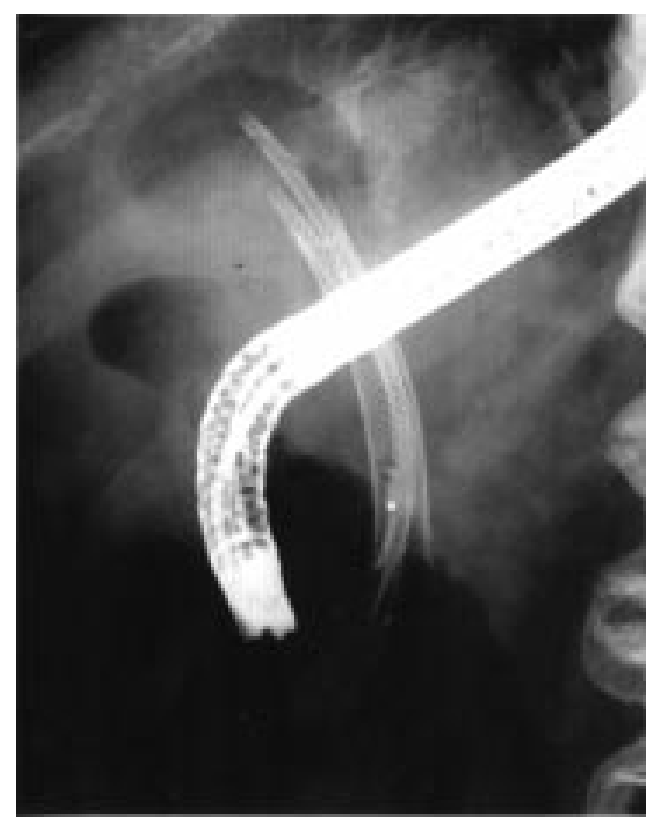

Figure 5 Two plastic stents inserted for occlusion of a Wallstent. 


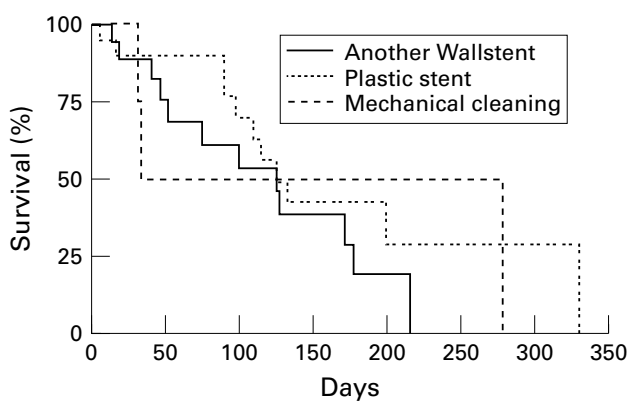

Figure 6 Overall patient survival following treatment of the first Wallstent occlusion.

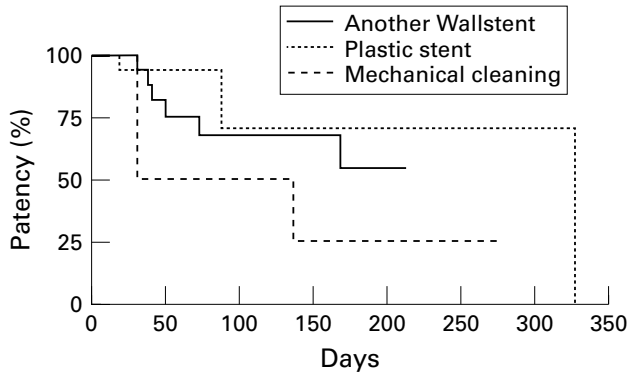

Figure 7 Cumulative patency of Wallstent following treatment of the occlusion.

respectively. There was no statistical difference in stent patency between the different treatments.

There was a positive correlation $(r=0.63$, $p=0.04$ ) between the patency of the first Wallstent and the period of patency of the second procedure (another Wallstent, plastic stent, or mechanical cleaning).

The mean number of ERCPs per patient was 1.31 in the second Wallstent group, 1.44 in the plastic stent group, and 1.4 in the mechanical cleaning group. The incremental cost of placing a second Wallstent $(\$ 995(£ 622))$ versus plastic stents $(\$ 83(£ 52))$ was $\$ 912$ $(£ 570)$. The cost effectiveness ratio of a second Wallstent versus plastic stent was therefore $912 /(1.44-1.31)=\$ 7015(£ 4384)$ per prevented ERCP. The incremental cost of placement of a second Wallstent versus mechanical cleaning ( $\$ 115(£ 72)$ ) was $\$ 880(£ 550)$. The cost effectiveness ratio of a second Wallstent versus mechanical cleaning was 880/ $(1.4-1.31)=\$ 9778(£ 6111)$ per prevented ERCP. The incremental cost of a plastic stent versus mechanical cleaning was $32 /(1.44-1.4)$ $=\$ 800(£ 500)$ per prevented ERCP. Thus, placement of a second Wallstent becomes economical only if ERCP related costs exceed $\$ 7015$ or $\$ 9778$ compared with plastic stents and mechanical cleaning respectively. Placement of a plastic stent compared with mechanical cleaning becomes economical when ERCP related costs exceed $\$ 800$.

\section{Discussion}

Most reports of the management of occluded Wallstents used in the biliary tree have either been case reports ${ }^{5}$ or a small part of a larger series evaluating other primary endpoints in the efficacy of Wallstents. ${ }^{27}$ In addition, long term results have not been published for placement of a second Wallstent or mechanical cleaning. Our retrospective study is the first to compare the different methods of treating Wallstent occlusion and also has the largest number of patients with occluded Wallstents in one series. We found no significant differences in the duration of patency or survival after managing Wallstent occlusions by insertion of another Wallstent, plastic stent, or mechanical cleaning. Thus all these methods of treatment appear to be equally effective. As median survival of patients following management of Wallstent occlusion was three months or less, the least costly method of treatment was that of a plastic stent as the insertion of another Wallstent did not seem to offer the advantage of longer patency. This is confirmed by incremental cost effectiveness analysis. As our study was retrospective and the subjects not randomly allocated, firm conclusions cannot be drawn until a prospective randomised and stratified study in larger numbers confirms our findings. However data from such a study are unlikely to be available for some time.

The median duration of patency of the primary Wallstent in our study was 3.5 months which is less than the $8-10$ months in previously reported studies. ${ }^{127}$ This may be due to a selection bias in our series which studied only patients whose Wallstents became occluded. The duration of patency of other Wallstents which did not occlude in the patients' lifetime was not included.

An incremental cost effectiveness analysis was performed because the three treatments for Wallstent occlusion differed only with respect to the equipment price as all appeared to be equally effective. Although ERCP related costs vary between different countries, it is apparent that the insertion of another Wallstent for an occluded Wallstent is the least cost effective option. The most cost effective option appears to be insertion of a plastic stent within the Wallstent. However in countries where ERCP costs are less than $\$ 800$, mechanical cleaning can be equally cost effective.

The major cause of Wallstent occlusion was tumour ingrowth in 28 of 44 occlusions (64\%), confirming previous observations. ${ }^{12}$ This problem may be overcome by the development of a silicone covering ${ }^{8}$; or the emergence of newer metallic stents which do not have an open framework, ${ }^{9}$ but additional problems may ensue, including stent migration and impairment of branch duct drainage in hilar lesions as well as potential obstruction of the pancreatic and cystic duct orifices.

The median patency of the second Wallstent within a Wallstent is relatively short at only 75 days which is less than that of the first Wallstent (102 days). The lack of prolonged patency following the second Wallstent insertion is one of the reasons why it has little advantage over insertion of a plastic stent. This may be because the majority of Wallstent occlusions are due to tumour ingrowth, and placement of a second Wallstent within the first Wallstent does not prevent this problem from recurring. On the other hand, a plastic stent within a Wallstent may prevent reocclusion due to tumour 
ingrowth although the plastic stent itself is prone to sludge encrustation. ${ }^{10}$

The positive correlation between the period of patency of the first Wallstent and the period of patency following treatment of the occlusion suggests that patients with early stent occlusion will occlude early following the second procedure. Furthermore, patients whose Wallstents remain patent for a long time may also have a longer patency following treatment of the occlusion. It could be argued that patients with early Wallstent occlusion may be selected for a subsequent cheaper procedure such as a plastic stent while patients with longer Wallstent patency might do better with another Wallstent. To determine whether such a strategy could be cost effective would require a large prospective randomised study.

Although there appeared to be a trend for a lower stent patency and survival following mechanical cleaning to treat a Wallstent occlusion, this was not statistically significant. However the number of patients in the group who had mechanical cleaning is small and larger numbers may be required to confirm this.

Alternative methods for treating Wallstent occlusions such as diathermy ${ }^{6}$ or hot tip laser probes ${ }^{11}$ have been described but these appear to carry an unnecessary risk of bleeding, ductal perforation, and stent fragmentation. ${ }^{12}$ In contrast none of the patients in our study had any complications following treatment of their occluded Wallstents.

In conclusion, although all three methods are equally effective in managing an occluded Wallstent, insertion of a plastic stent within a Wallstent appears to be the most cost effective method; in some health care environments, mechanical cleaning may be as cost effective. A prospective randomised stratified study is required to confirm this.

T C K Tham was supported as a Visiting Fellow by a grant from the Northern Ireland Council for Postgraduate Medical Education, J Vandervoort by a grant from OLV Hospital, Belgium, and both by a grant from Fujinon Photo Optical, Inc., Wayne, New Jersey and Omiya City, Japan. We are grateful to Dr Neil McDougall and David Gill of the Department of Medicine, the Queen's University of Belfast, for assistance with graphical and statistical analysis.

1 Carr-Locke DL, Ball TJ, Connors PJ, et al. Multicenter randomized trial of Wallstent biliary endoprosthesis versus plastic stents [abstract]. Gastrointest Endosc 1993;39:A310.

2 Davids PHP, Groen AK, Rauws EAJ, et al. Randomized trial of self-expanding metal stents versus polyethylene stents for distal malignant biliary obstruction. Lancet 1992;340: 1488-92.

3 Cotton PB, Lehman G, Vennes J, et al. Endoscopic sphincterotomy complications and their management: an attempt at consensus. Gastrointest Endosc 1991;37:383-93.

4 Detsky AS, Naglie IG. A clinician's guide to costeffectiveness analysis. Ann Intern Med 1990;113:147-54.

5 Mixon T, Goldschmid S, Brady PG, et al. Endoscopic management of expandable metallic biliary stent occlusion. agement of expandable metallic

6 Cremer M, Deviere J, Sugai B, et al. Expandable biliary metal stents for malignancies: endoscopic insertion and diathermic cleaning for tumor ingrowth. Gastrointest Endosc 1990;36:451-7.

7 Schmassmann A, von Gunten E, Knuchel J, et al. Wallstents versus plastic stents in malignant biliary obstruction: effects of stent patency of the first and second stent on patient compliance and survival. Am $\mathcal{f}$ Gastroenterol 1996;91:654-9.

8 Silvis SE, Sievert CE, Vennes JA, et al. Comparison of covered vs uncovered wire mesh stents in the canine biliary tract. Gastrointest Endosc 1994;40:17-21.

9 Goldin E, Beyar M, Safra T, et al. A new self-expandable and removable metal stent for biliary obstruction-a and removable metal stent for biliary obst

10 Speer AG, Cotton PB, Rode J, et al. Biliary stent blockage with bacterial biofilm: a light and electron microscopy study. Ann Intern Med 1988;108:546-53.

11 Loseff SV, Druy E, Jelinger E, et al. Use of hot-tip laser probes to recanalize occluded expandable metallic biliary endoprostheses. AfR Am F Roentgenol 1992;158:199-201.

12 Ell C, Fleig WE, Hochberger J. Broken biliary metal stent after repeated electrocoagulation for tumor ingrowth. Gastrointest Endosc 1992;38:197-9. 